\title{
ON NON-ZERO VALUES OF THE CENTRAL HOMOLOGICAL DIMENSION OF $C^{*}$-ALGEBRAS
}

\author{
by Z. A. LYKOVA
}

(Received 23 July, 1991)

The paper is related to the area which was recently called topological homology $[3,6,12,16,4]$. We consider questions associated with the central Hochschild cohomology of $\mathrm{C}^{*}$-algebras. The study of the latter was begun by J. Phillips and I. Raeburn in $[9,10]$, when they were investigating some problems of the theory of perturbations of $\mathrm{C}^{*}$-algebras. In [8] we obtained a description of the structure of $\mathrm{C}^{*}$-algebras with central bidimension zero: it was proved that these $C^{*}$-algebras are unital and have continuous trace. In the special case of separable and a priori unital $\mathrm{C}^{*}$-algebras this statement was proved by J. Phillips and I. Raeburn in [11] with the help of a different approach. The question was raised. Which values can the central bidimension of $\mathrm{C}^{*}$-algebras take? In the present paper it is shown that, for any CCR-algebra $A$ having at least one infinitedimensional irreducible representation, the central bidimension and the global central homological dimension of $A$ are greater than one. At the same time it is proved that there exist CCR-algebras which are centrally biprojective, but which have both dimensions equal to one. This situation contrasts with the state of affairs in the "traditional" theory of the Banach Hochschild cohomology. Recall [3, Ch. 5] that the bidimension and the global homological dimension of any infinite-dimensional biprojective $\mathrm{C}^{*}$-algebra are equal to two. Besides, there is no CCR-algebra of bidimension one (respectively, global homological dimension one). See [7].

One can see the main concepts of the relative homological theory over a commutative Banach algebra $B$ in [8]. In Section 0 , we recall some of them which will be used in the present paper. In Section 1, sufficient conditions are given which imply the relative homological dimensions of a Banach algebra over $B$ are equal to one. In Section 2 , we calculate the central bidimension and the global central homological dimension of biprojective $\mathrm{C}^{*}$-algebras and study some structural properties of central biprojective $C^{*}$-algebras. In Section 3, a lower estimate of the global central homological dimension of CCR-algebras which have infinite-dimensional irreducible representations will be obtained.

0. Necessary information. Let $B$ be a commutative Banach algebra with an identity $e_{B}$. A Banach algebra $A$ is called a Banach $B$-algebra if $A$ is a Banach $B$-module such that for any $a_{1}, a_{2} \in A$, and $b \in B$ the condition $\left(a_{1} a_{2}\right) . b=a_{1}\left(a_{2} \cdot b\right)=\left(a_{1} \cdot b\right) a_{2}$ holds. (For a purely algebraic approach to the theory of $B$-algebras, see $[1, C h$. 9].) Here and everywhere afterwards "a Banach $B$-module" will mean a unital symmetrical Banach $B$-bimodule. Simultaneously with a Banach $B$-algebra $A$ we consider a Banach $B$-algebra $A_{B}=A \oplus B$ with operations

$$
\begin{gathered}
\left(a_{1}, b_{1}\right)\left(a_{2}, b_{2}\right)=\left(a_{1} a_{2}+a_{1} . b_{2}+b_{1}, a_{2}, b_{1} b_{2}\right), \\
b .\left(a_{1}, b_{1}\right)=\left(b . a_{1}, b b_{1}\right)=\left(a_{1}, b_{1}\right) . b,\left(\left(a_{1}, b_{1}\right),\left(a_{2}, b_{2}\right) \in A_{B}, b \in B\right),
\end{gathered}
$$

and a norm which makes $A_{B}$ into a Banach algebra such that $\|(a, 0)\|=\|a\|(a \in A)$, and $\|(0, b)\|=\|b\|(b \in B)$. In particular, we can take $\|(a, b)\|=\max \{\|a\|,\|b\|\}$. Recall (see,

Glasgow Math. J. 34 (1992) 369-378. 
for example, [8]), that a left (right, bi-) Banach $A$-module $X$ is called the left (right, bi-) Banach $(A, B)$-module if, in addition, $X$ is a Banach $B$-module and for any $a \in A, b \in B$ and $x \in X$ the following conditions $(a . x) . b=a .(x . b)=(a . b) . x$ (respectively, or (and) $(x, a) \cdot b=x .(a \cdot b)=(x, b) \cdot a)$ hold. A morphism of left (right, bi-) Banach $A$-modules is called a morphism of left (respectively, right, bi-) Banach $(A, B)$-modules if, in addition, it is a morphism of Banach $B$-modules. The category of left Banach $(A, B)$-modules and their morphisms is denoted by $(A, B)$-mod; $(A, B)$-mod- $(A, B)$ denote the corresponding category of Banach $(A, B)$-bimodules. For $X, Y \in(A, B)$-mod $((A, B)$-mod- $(A, b))$ the Banach space of morphisms from $X$ to $Y$ is denoted by $(A, B) h(X, Y)$ (respectively, $(A, B) h_{(A, B)}(X, Y)$ ). We denote by $A_{+}$the Banach algebra obtained by adjoining an identity $e_{+}$to $A$.

We note that any Banach $(A, B)$-module $X$ is a Banach $\left(A_{B}, B\right)$-module with operations of external multiplications $(a, b) \cdot x=a \cdot x+b \cdot x$ or (and) $x \cdot(a, b)=x \cdot a+x \cdot b$ $\left((a, b) \in A_{B}, x \in X\right)$, and the same $B$-module structure.

In the homological theory of Banach $B$-algebras the Banach $B$-cohomology of Hochshild $H_{B}^{n}(A, X)$ of the Banach $B$-algebra $A$ with coefficients in a $(A, B)$-bimodule $X$ is of great interest.

DEFINITION 0.1. Let

$$
0 \rightarrow C_{B}^{0}(A, X) \stackrel{\delta^{0}}{\longrightarrow} \ldots \stackrel{\delta^{n-1}}{\longrightarrow} C_{B}^{n}(A, X) \stackrel{\delta^{n}}{\longrightarrow} C_{B}^{n+1}(A, X) \stackrel{\delta^{n+1}}{\longrightarrow} \ldots,\left(\mathscr{C}_{B}(A, X)\right)
$$

be a complex, where $C_{B}^{0}(A, X)$ is the $B$-module $X$ and, for $n>0, C_{B}^{n}(A, X)$ is the $B$-module of all continuous $n-B$-linear maps $f: A \times A \times \ldots \times A \rightarrow X$. The connecting maps $\delta^{n}(n=0,1, \ldots)$ act by the formulae $\left(\delta^{0} x\right)(a)=a . x-x . a$ and for, $n>0$, we have

$$
\begin{aligned}
& \left(\delta^{n} f\right)\left(a_{1}, \ldots, a_{n+1}\right)=a_{1}, f\left(a_{2}, \ldots, a_{n+1}\right) \\
& \quad+\sum_{i=1}^{n}(-1)^{i} f\left(a_{1}, \ldots, a_{i} a_{i+1}, \ldots, a_{n+1}\right)+(-1)^{n+1} f\left(a_{1}, \ldots, a_{n}\right) \cdot a_{n+1} .
\end{aligned}
$$

This complex is called the standard $B$-cohomological complex. The $n$-th cohomology of $\mathscr{C}_{B}(A, X)$ is called the $n$-dimensional $B$-cohomology group of $A$ with coefficients in $X$ and is denoted by $H_{B}^{n}(A, X)$.

The $B$-bidimension of the Banach $B$-algebra $A$ is a number (or $\infty$ )

$$
d b_{B} A=\inf \left\{n: H_{B}^{n+1}(A, X)=0 \text { for all } X \in(A, B) \text {-mod- }(A, B)\right\} .
$$

Homological characteristics are closely connected with the concept of projectivity. A Banach $(A, B)$-module $P$ is called $(A, B)$-projective if, for any Banach $(A, B)$-module $Y$, any epimorphism $\varphi$ from $Y$ onto $P$ such that $\varphi$ has a right inverse morphism of Banach $B$-modules must have also a right inverse morphism of Banach $(A, B)$-modules.

A Banach $B$-algebra $A$ is called $B$-biprojective if it is projective as a Banach $(A, B)$-bimodule.

For $X \in(A, B)-\bmod ((A, B)-\bmod -(A, B))$ a complex over $X$

$$
0 \longleftarrow X \stackrel{\varepsilon}{\longleftarrow} P_{0} \stackrel{\varphi_{0}}{\longleftarrow} P_{1} \stackrel{\varphi_{1}}{\longleftarrow} P_{2} \stackrel{\varphi_{2}}{\longleftarrow} \ldots(0 \longleftarrow X \longleftarrow \mathscr{P})
$$


is called a projective resolution if it is splittable regarded as a complex of Banach $B$-module and all $P_{i}(i=1,2, \ldots)$ are projective in $(A, B)$-mod (respectively, in $(A, B)$-mod- $(A, B))$. We shall denote the $n$-th cohomology of the complex $h_{(A, B)}(\mathscr{P}, Y)$ by $\operatorname{Ext}_{(A, B)}^{n}(X, Y)(X, Y \in(A, B)$-mod) and the $n$-th cohomology of the complex ${ }_{(A, B)} h_{(A, B)}(\mathscr{P}, Y)$ by ${ }_{(A, B)} \operatorname{Ext}_{(A, B)}^{n}(X, Y)(X, Y \in(A, B)-\bmod -(A, B))$.

If $P_{i} \neq 0$ for $i=1,2, \ldots, n$ and $P_{i}=0$ for $i>n$, we say that the $(A, B)$-projective resolution $0 \leftarrow X \leftarrow \mathscr{P}$ has a length $n$. The length of the shortest $(A, B)$-projective resolution is called the $B$-homological dimension of the $(A, B)$-module $X$, and it is denoted by $d h_{\mathscr{C}} X$, where $\mathscr{K}$ is $(A, B)$-mod or $(A, B)$-mod- $(A, B)$, respectively. We note that

$$
d h_{\mathscr{K}} X=\inf \left\{n: \operatorname{Ext}_{\mathscr{K}}^{n+1}(X, Y)=0 \text { for all } Y \in \mathscr{K}\right\} .
$$

The global $B$-homological dimension of a Banach $B$-algebra $A$ is defined as a number $($ or $\infty)$

$$
d g_{B} A=\sup \left\{d h_{(A, B)} X: X \in(A, B)-\bmod \right\} .
$$

In [8] it was proved that $d g_{B} A \leqslant d b_{B} A$.

In the case $B=\mathbb{C}$, where we come to the well-known definitions of the homology of Banach algebras [3], we omit " $B$ ".

Recall that in [8] it was shown that, for any Banach $B$-algebra $A$, up to a topological isomorphism, $H_{B}^{n}(A, X)=\operatorname{Ext}_{(A, B)-(A, B)}^{n}\left(A_{B}, X\right)$, where $A_{B}$ is an $(A, B)$-bimodule with the following structures of left and right $A$-modules and $B$-module:

$$
\begin{aligned}
& a \cdot\left(a_{1}, b_{1}\right)=(a, 0)\left(a_{1}, b_{1}\right) ;\left(a_{1}, b_{1}\right) \cdot a=\left(a_{1}, b_{1}\right)(a, 0), \\
& b .\left(a_{1}, b_{1}\right)=\left(b . a_{1}, b b_{1}\right)=\left(a_{1}, b_{1}\right) . b\left(a \in A,\left(a_{1}, b_{1}\right) \in A_{B}, b \in B\right) .
\end{aligned}
$$

The symbol $\bigotimes_{B}$ will denote the projective tensor product of Banach $B$-modules [13]. The identity operator is denoted by $1_{X}: X \rightarrow X: x \mapsto x$.

\section{The $B$-homological dimensions of some $B$-biprojective algebras.}

Proposition 1.1. Let $A$ be a B-biprojective Banach algebra without a right identity. Suppose that the morphism of diagonal inclusion

$$
\Delta: A \hat{\bigotimes}_{B} A \rightarrow\left(A_{B} \hat{\bigotimes}_{B} A\right) \oplus\left(A \hat{\bigotimes}_{B} A_{B}\right): a \otimes b \mapsto((a, 0) \otimes b, a \otimes(b, 0))
$$

has a left inverse morphism in $(A, B)$-mod- $(A, B)$. Then $d b_{B} A=d g_{B} A=1$.

Proof. First we note that there exists an $(A, B)$-projective resolution of the trivial left $(A, B)$-module $B$ of the form

$$
0 \leftarrow B \leftarrow A_{B} \leftarrow \text { i } A \leftarrow 0,
$$

where $i$ is the morphism of inclusion. If $d h_{(A, B)} B=0$, then there exists a morphism of left Banach $(A, B)$-modules $j: A_{B} \rightarrow A$ such that $j \circ i=1_{A}$, and, consequently, $A$ has a right identity $e_{1}=j\left(\left(0, e_{B}\right)\right)$. The contradiction shows that $d h_{(A, B)} B=1$. Thus, the following inequalities hold:

$$
1=d h_{(A, B)} B \leqslant d g_{B} A \leqslant d b_{B} A \text {. }
$$


Now we consider an analogue of entwining resolution from $[3,5.2 .1]$ where $i$ is the morphism of inclusion and $m$ is the product morphism. It is easy to prove the splittability of the given complex in the category of Banach $B$-modules. Besides, we note that all Banach $(A, B)$-bimodules participating in the resolution (1) are $(A, B)$-projective.

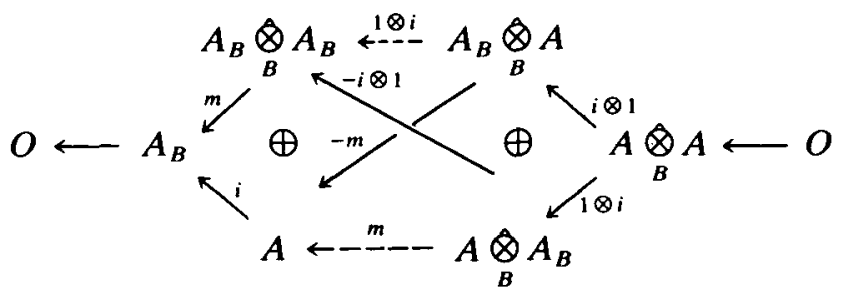

Furthermore, we consider the short splittable complex in the category of Banach $B$-modules complexes provided by (1):

and

$$
0 \longleftarrow A_{B} \stackrel{\partial-1}{\longleftarrow}\left(A_{B} \hat{\bigotimes}_{B} A_{B}\right) \oplus A \longleftarrow W \longleftarrow 0
$$

$$
0 \longleftarrow W \stackrel{\partial_{0}}{\longleftarrow}\left(A_{B} \hat{\bigotimes}_{B} A\right) \oplus\left(A \hat{\bigotimes}_{B} A_{B}\right) \stackrel{\partial_{1}}{\longleftarrow} A \hat{\bigotimes}_{B} A \longleftarrow 0,
$$

where $W=\operatorname{Ker} \partial_{-1}=\operatorname{Im} \partial_{0}$, and the morphisms $\partial_{i}(i=-1,0,1)$ are defined naturally by the morphisms indicated on the diagram (1).

Now, by the condition of the proposition, there exists a morphism of Banach $(A, B)$-bimodules $\rho:\left(A_{B} \hat{\otimes}_{B} A\right) \oplus\left(A \hat{\otimes}_{B} A_{B}\right) \rightarrow A \hat{\bigotimes}_{B} A$ such that $\rho \circ \partial_{1}=1_{A \hat{\otimes}_{B} A}$. Hence the resolution $(3)$ is splittable in $(A, B)$-mod- $(A, B)$, and, consequently, $W$ is an $(A, B)$-projective Banach $(A, B)$-bimodule. Then the complex (2) is the $(A, B)$-projective resolution of length one in $(A, B)$-mod- $(A, B)$. So, in virtue of Lemma 2.2 of [8],

$$
d b_{B} A=d h_{(A, B)-(A, B)} A_{B} \leqslant 1 .
$$

Thus, we have

$$
1=d h_{(A, B)} B \leqslant d g_{B} A \leqslant d b_{B} A=d h_{(A, B)-(A, B)} A_{B} \leqslant 1 .
$$

THEOREM 1.1. Let $A$ be a commutative Banach algebra with an identity, and let I be a proper closed ideal of $A$ with a bounded approximate identity. Then, for $B=A$,

$$
d g_{B} I=d b_{B} I=1 .
$$

Proof. By analogy with Theroem 3.24 of $[4$, Ch. 6], up to an isomorphism of Banach $(I, B)$-bimodules,

$$
I=I_{B} \hat{\bigotimes}_{B} I \hat{\bigotimes}_{B} I_{B}: a \mapsto \lim _{v} \lim _{\mu}\left[(a, 0) \otimes e_{v} \otimes\left(e_{\mu}, 0\right)\right],
$$

where $e_{v}(v \in \Lambda)$, is a bounded approximate identity in $I$. Hence $I$ is a $B$-biprojective $B$-algebra. 
Besides, the morphism of Banach $(I, B)$-bimodules $\rho=(\varphi \oplus 0)$ is a left inverse to the morphism of the diagonal inclusion

$$
\Delta: I \hat{\bigotimes}_{B} I \rightarrow\left(I_{B} \hat{\bigotimes}_{B} I\right) \oplus\left(I \hat{\bigotimes}_{B} I_{B}\right): a \otimes b \mapsto((a, 0) \otimes b, a \otimes(b, 0)),
$$

where $\varphi:\left(I_{B} \hat{\bigotimes}_{B} I\right) \rightarrow I \hat{\bigotimes}_{B} I:\left(a_{1}, b_{1}\right) \otimes a_{2} \mapsto \lim _{v}\left(a_{1} a_{2}+b_{1} a_{2}\right) \otimes e_{v}$ is a morphism of Banach $(I, B)$-bimodules. Thus the conditions of Proposition 1.1 are satisfied and consequently, $d g_{B} I=d b_{B} I=1$.

2. On the central homological dimensions of biprojective $\mathbf{C}^{*}$-algebras. Let $Z\left(A_{+}\right)$ be the centre of $A_{+}$. For $B=Z\left(A_{+}\right)$, a $B$-biprojective Banach $B$-algebra $A$ will be called centrally biprojective.

Proposition 2.1. Let $A$ be a centrally biprojective $C^{*}$-algebra. Then all its irreducible representations are finite-dimensional.

We begin the proof of this statement with the next lemma.

LEMMA 2.1. Let $A$ be a centrally biprojective Banach algebra with a bounded approximate identity $e_{v}(v \in \Lambda)$, let $M$ be a closed two-sided ideal of $A$, and let $\varphi: A \rightarrow A / M$ be the natural epimorphism. Then there exists a morphism of Banach $\left(A / M, Z\left((A / M)_{+}\right)\right)$-bimodules given by

$$
\tilde{\rho}: A / M \rightarrow(A / M)_{+} \hat{\bigotimes}_{\left((A / M)_{+}\right)} A / M
$$

such that the equality $\tilde{\pi}_{+}{ }^{\circ} \tilde{\rho}=1_{A / M}$ holds, where

$$
\tilde{\pi}_{+}:(A / M)_{+} \hat{\bigotimes}_{Z\left((A / M)_{+}\right)} A / M \rightarrow A / M: \bar{a} \otimes \bar{b} \mapsto \bar{a} \cdot \bar{b}
$$

is the canonical morphism.

Proof. Since $A$ is projective in $\left(A, Z\left(A_{+}\right)\right)$-mod- $\left(A, Z\left(A_{+}\right)\right)$, there exists a morphism of Banach $\left(A, Z\left(A_{+}\right)\right)$-bimodules $\rho: A \rightarrow A_{+} \hat{\bigotimes}_{Z\left(A_{+}\right)} A$ such that $\pi_{+}{ }^{\circ} \rho=1_{A}$, where

$$
\pi_{+}: A_{+} \bigotimes_{Z\left(A_{+}\right)} A \rightarrow A: a \otimes b \mapsto a . b .
$$

We define a morphism of Banach $\left(A / M, Z\left(\left(A / M_{+}\right)\right)\right.$-bimodules by

$$
\tilde{\rho}: A / M \rightarrow(A / M)_{+} \hat{\bigotimes}_{Z\left((A / M)_{+}\right)} A / M
$$

putting, for $\bar{a}=a+M$ in $A / M, \tilde{\rho}(a+m)=(\tilde{\varphi} \otimes \varphi) \rho(a)$, where $\tilde{\varphi}: a_{+} \rightarrow(A / M)_{+}$: $a+\lambda e_{+} \mapsto \varphi(a)+\lambda e_{+}$. The morphism $\tilde{\rho}$ is well-defined since, for $u \in M$,

$$
\begin{aligned}
\tilde{\rho}(u+M) & =(\tilde{\varphi} \otimes \varphi) \rho(u)=(\tilde{\varphi} \otimes \varphi) \rho\left(\lim _{v} u e_{v}\right) \\
& =\lim _{v}(\tilde{\varphi} \otimes \varphi) \rho\left(u e_{v}\right)=\lim _{v} \varphi(u) \cdot(\tilde{\varphi} \otimes \varphi) \rho\left(e_{v}\right)=0 .
\end{aligned}
$$


Besides,

$$
\left(\tilde{\pi}_{+} \circ \tilde{\rho}\right)(a+M)=\left(\tilde{\pi}_{+} \circ(\tilde{\varphi} \otimes \varphi)\right) \rho(a)=\varphi\left(\pi_{+} \rho(a)\right)=a+M .
$$

Conclusion of the proof of Proposition 2.1. Let $T$ be an infinite-dimensional, irreducible representation of $A$ on a Hilbert space $H T: A \rightarrow \mathscr{B}(H)$, where $\mathscr{B}(H)$ is the $C^{*}$-algebra of continuous linear operators on $H$. By Lemma 2.4 .4 of [14], the commutant of $T(A)$ in $\mathscr{B}(H)$ is isomorphic to $\mathbb{C}$. Denote by $I$ the primitive ideal $\operatorname{Ker} T$. Then it is easy to see that the centre of $A / I$ is isomorphic to $\mathbb{C}$ or $(0)$. Recall $([2,1.7 .2])$ that each $C^{*}$-algebra has a bounded approximate identity. Therefore, in virtue of Lemma 2.1 , there exists a morphism of Banach $A / I$-bimodules $\tilde{\rho}: A / I \rightarrow(A / I)_{+} \hat{\otimes} A / I$ such that $\tilde{\pi}_{+}{ }^{\circ} \tilde{\rho}=1_{A / I}$. Hence we can show that there exists a morphism of Banach $A / I$-bimodules $\tilde{\rho}: A / I \rightarrow A / I \hat{\otimes} A / I$ such that $\tilde{\pi} \circ \tilde{\rho}=1_{A / I}$ (because $A / I$ has a bounded approximate identity). Hence $A / I$ is biprojective.

By the structural Theorem 5.5 of [15] (see also [3, 4.5.15]), a biprojective $C^{*}$-algebra is a $c_{0}$-sum of some family of full matrix $C^{*}$-algebras $([12,1.4 .15])$, and, consequently, $A / I$ is a full matrix $C^{*}$-algebra and $I$ is a maximal closed two-sided ideal.

Recall [3, Ch. 5] that the homological dimensions of an infinite-dimensional biprojective $C^{*}$-algebra $A$ satisfy the equality $d b A=d g A=2$. Note also that each biprojective $\mathrm{C}^{*}$-algebra is $Z\left(A_{+}\right)$-biprojective, but, as will be shown later, its central homological dimensions are equal to one.

TheOREM 2.1. Let $A$ be a $c_{0}$-sum of some family of full matrix $C^{*}$-algebras. If $A$ is finite-dimensional, then $d b_{Z\left(A_{+}\right)} A=d g_{Z\left(A_{+}\right)} A=0$. If $A$ is infinite-dimensional, then $d b_{Z\left(A_{+}\right)} A=d g_{Z\left(A_{+}\right)} A=1$.

Proof. Recall that $A$ is biprojective ([15]), and, consequently, is $Z\left(A_{+}\right)$-biprojective.

In view of the main theorem of [8], a $C^{*}$-algebra $A$ with the zero central bidimension is a $C^{*}$-algebra with a continuous trace and an identity. Hence, for the case in which $A$ is finite-dimensional, we obtain that

$$
0=d h_{\left(A, Z\left(A_{+}\right)\right)} A \leqslant d g_{Z\left(A_{+}\right)} A \leqslant d b_{Z\left(A_{+}\right)} A=0 .
$$

When $A$ is infinite-dimensional, we see that $A$ does not have a right identity. Besides, now we shall prove that the morphism of the diagonal inclusion

$$
\Delta: A \hat{\bigotimes}_{B} A \rightarrow\left(A_{B} \hat{\bigotimes}_{B} A\right) \oplus\left(A \hat{\bigotimes}_{B} A_{B}\right)
$$

has a left inverse morphism in $(A, B)$-mod- $(A, B)$, where $B=Z\left(A_{+}\right)$.

The condition of the theorem implies that $A$ is a $c_{0}$-sum of some family of full matrix $\mathrm{C}^{*}$-algebras $\left\{A_{v}: v \in \Lambda\right\}$. We shall denote by $N(\Lambda)$ the set of finite subsets of $\Lambda$, ordered by inclusion. Let $\lambda \in N(\Lambda)$. Before we define $\rho$, we put

$$
\varphi: A_{B} \times A \rightarrow A \hat{\bigotimes}_{B} A:\left(\left(a_{1}, b_{1}\right), a_{2}\right) \mapsto \lim _{\lambda} \sum_{\alpha \in \lambda}\left(a_{1} e_{\alpha}+b_{1}, e_{\alpha}\right) \otimes e_{\alpha} a_{2}
$$

where $e_{\alpha}(v)=\delta_{\alpha}^{v} e_{A_{v}}, \delta_{\alpha}^{v}$ is the Kronecker symbol, and $e_{A_{v}}$ is the identity matrix from $A_{v}$. Note that, for $a_{1}, a_{2} \in A$ and $b_{1}, b_{2} \in B$, the equality $\varphi\left(\left(a_{1}, b_{1}\right) \cdot b, a_{2}\right)=\varphi\left(\left(a_{1}, b_{1}\right), b \cdot a_{2}\right)$ 
holds. Besides with the help of a known method for the estimate of the norm of elements in $A \hat{\otimes} A([4,0.3 .30])$, we can show that

$$
\left\|\sum_{i=1}^{m}\left(a_{1} e_{\alpha_{i}}+b_{1}, e_{\alpha_{i}}\right) \otimes e_{\alpha_{i}} a_{2}\right\| \leqslant 1 / m \sum_{k=1}^{m}\left\|\sum_{i=1}^{m} \zeta^{k(i-1)}\left(a_{1} e_{\alpha_{i}}+b_{1} \cdot e_{\alpha_{i}}\right)\right\|\left\|\sum_{l=1}^{m} \zeta^{k(l-1)} e_{\alpha_{l}} a_{2}\right\|,
$$

where $\zeta$ is a primitive $m$-th degree root of the identity from $\mathbb{C}$. Therefore, since the inequalities

$$
\left\|\sum_{i=1}^{m} \xi^{k(i-1)}\left(a_{1} e_{\alpha_{i}}+b_{1} \cdot e_{\alpha_{i}}\right)\right\| \leqslant \sup _{1 \leqslant i \leqslant m}\left\|a_{1} e_{\alpha_{i}}+b_{1} \cdot e_{\alpha_{i}}\right\|
$$

and

$$
\left\|\sum_{l=1}^{m} \zeta^{k(l-1)} e_{\alpha_{l}} a_{2}\right\| \leqslant \sup _{1 \leqslant l \leqslant m}\left\|e_{\alpha_{l}} a_{2}\right\|
$$

hold, we can see that

$$
\left\|\sum_{i=1}^{m}\left(a_{1} e_{\alpha_{i}}+b_{1} \cdot e_{\alpha_{i}}\right) \otimes e_{\alpha_{i}} a_{2}\right\| \leqslant \sup _{1 \leqslant i \leqslant m}\left\|a_{1} e_{\alpha_{i}}+b_{1} \cdot e_{\alpha_{i}}\right\| \sup _{1 \leqslant / \leqslant m}\left\|e_{\alpha_{1}} a_{2}\right\| .
$$

Since $a_{2} \in A$, for each $\varepsilon>0$ there exists $\lambda_{0} \in N(\Lambda)$ such that $\sup _{\mu \notin \lambda_{1}}\left\|e_{\mu} a_{2}\right\|<\varepsilon$, and, consequently, for all $\lambda^{\prime} \geqslant \lambda_{0}$ and $\lambda^{\prime \prime} \geqslant \lambda_{0}$ in $N(\Lambda)$, we have

$$
\begin{aligned}
\| \sum_{\alpha \in \lambda^{\prime}}\left(a_{1} e_{\alpha}+b_{1} \cdot e_{\alpha}\right) \otimes e_{\alpha} a_{2}-\sum_{\alpha \in \lambda^{\prime \prime}}\left(a_{1} e_{\alpha}\right. & \left.+b_{1} . e_{\alpha}\right) \otimes e_{\alpha} a_{2} \| \\
& \leqslant \sup _{\alpha \notin \lambda_{0}}\left\|a_{1} e_{\alpha}+b_{1} . e_{\alpha}\right\| \sup _{\alpha \notin \lambda_{0}}\left\|e_{\alpha} a_{2}\right\|<2 \varepsilon\left\|\left(a_{1}, b_{1}\right)\right\| .
\end{aligned}
$$

Therefore $u_{\lambda}=\lim _{\lambda} \sum_{\alpha \in \lambda}\left(a_{1} e_{\alpha}+b_{1} . e_{\alpha}\right) \otimes e_{\alpha} a_{2}$ converges in $A \hat{\bigotimes_{B}} A$ and $\|\varphi\| \leqslant 2$. Then, in virtue of the universal property of the projective tensor product ([13]), there exists a continuous linear operator $\tilde{\varphi}: A_{B} \hat{\bigotimes}_{B} A \rightarrow A \hat{\bigotimes}_{B} A$ such that $\|\tilde{\varphi}\| \leqslant 2$. We can verify that $\tilde{\varphi}$ is a morphism of $(A, B)$-bimodules.

We define $\rho=(\tilde{\varphi} \oplus 0)$. For $a_{1} \otimes a_{2}$ from $A \hat{\bigotimes}_{B} A$, we obtain

$$
\rho \circ \partial_{1}\left(a_{1} \otimes a_{2}\right)=\tilde{\varphi}\left(a_{1} \otimes a_{2}\right)=\lim _{\lambda} \sum_{\alpha \in \lambda}\left(a_{1} e_{\alpha} \otimes e_{\alpha} a_{2}\right)=\lim _{\lambda} \sum_{\alpha \in \lambda} a_{1} e_{\alpha} \otimes a_{2}=a_{1} \otimes a_{2} .
$$

Consequently, $\rho \circ \partial_{1}=1_{A \underset{B}{\hat{\otimes}_{A}}}$. Thus Proposition 1.1 implies that for an infinitedimensional $A$ we have $d g_{B} A=d b_{B} A=1$.

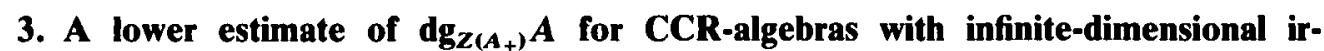
reducible representations. We know ([8]) that there exist CCR-algebras with $d b_{Z\left(A_{+}\right)} A=0$. These are algebras having a continuous trace and an identity. In the 
previous section we prove that infinite-dimensional CCR-algebras $A$ which are a $c_{0}$-sum of

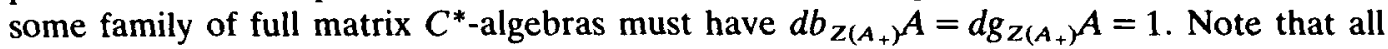
irreducible representations of these algebras are finite dimensional.

Theorem 3.1. Let $A$ be a CCR-algebra. Suppose that there exists an infinitedimensional, irreducible representation of this algebra. Then

$$
d b_{Z\left(A_{+}\right)} A \geqslant d g_{Z\left(A_{+}\right)} A \geqslant 2 .
$$

Proof. Let $T$ be an infinite-dimensional, irreducible representation of $A$ on a Hilbert space $H$. Then the $\mathrm{C}^{*}$-algebra $A / \operatorname{KerT}$ is isomorphic to the $\mathrm{C}^{*}$-algebra of compact operators $\mathscr{K}(H)([2,4.2 .5])$. In [7], it was shown that $d g \mathscr{K}(H) \geqslant 2$. Thus, for the proof of the theorem, it is sufficient to prove the following statement.

LeMMA 3.1. Let $A$ be a Banach algebra, and let I be a primitive ideal with a bounded

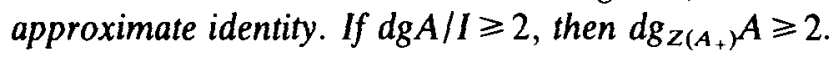

Proof. The proof of this lemma is similar to the proof of the lemma from [7]. Let $X$ be a left $A / l$-module such that $d h_{A / l} x \geqslant 2$. It is easy to see that the multiplication $a . x=\tau(a) . x(a \in A, x \in X)$, where $\tau: A \rightarrow A / I$ is a natural epimorphism, provides a structure of a left $A$-module. The structure of the $Z\left(A_{+}\right)$-module on $X$ is defined by the following formula:

$$
z \cdot x=x \cdot z=\tilde{\tau}(a) \cdot x, \text { where } \quad \tilde{\tau}: A_{+} \rightarrow(A / I)_{+}: a+\lambda e_{+} \mapsto \tilde{\tau}(a)+\lambda e_{+} .
$$

Suppose that $d h_{\left(A, Z\left(A_{+}\right)\right)} X \leqslant 1$. Then the left $\left(A, Z\left(A_{+}\right)\right)$-module $J=\operatorname{Ker} \pi_{+}$is projective, where $\pi_{+}: A_{Z\left(A_{+}\right)} \underset{Z\left(A_{+}\right)}{\otimes} X \rightarrow X: a \otimes x \mapsto a \cdot x$ is the canonical morphism.

First we consider the case where the algebra $A / I$ does not have an identity. By Theorem 1 of [5], the right $A_{Z\left(A_{+}\right)}$-modules $A_{Z\left(A_{+}\right)} / l$ and $A_{+}$are flat. Consequently, the right $A_{Z\left(A_{+}\right)}$-module $A_{+} / I \simeq A_{+} \underset{A_{Z\left(A_{+}\right)}}{\hat{A_{Z\left(A_{+}\right)}}} / l$ is also flat. Hence the sequence

$$
0 \leftarrow A_{+} / I \underset{A_{Z\left(A_{+}\right)}}{\hat{\bigotimes}} X \stackrel{1 \otimes \pi_{+}}{\longleftarrow} A_{+} / I \underset{A_{Z\left(A_{+}\right)}}{\bigotimes_{Z\left(A_{+}\right)}} A_{+} \underset{\bigotimes_{1}}{\otimes} X \longleftarrow A_{+} / I \underset{A_{Z\left(A_{+}\right)}}{\hat{\bigotimes}} J \longleftarrow 0
$$

is exact. Besides, in view of the Lemma of [5],

$$
A_{+} / I \underset{A_{Z\left(A_{+}\right)}}{\hat{\bigotimes}} A_{+} \underset{Z\left(A_{+}\right)}{\hat{\bigotimes}} X=A_{+} / I \underset{Z\left(A_{+}\right)}{\hat{\bigotimes}} X \text { and } A_{+} / I \underset{A_{Z\left(A_{+}\right)}}{\hat{\bigotimes}} X=X
$$

up to an isomorphism of left Banach $A / I$-modules. Thus, for the $A / I$-module $X$, there exists an admissible short exact sequence

$$
0 \leftarrow X \leftarrow A_{+} / I \underset{Z\left(A_{+}\right)}{\hat{\otimes}} X \leftarrow A_{+} / I \underset{A_{Z\left(A_{+}\right)}}{\hat{\otimes}} J \leftarrow 0 .
$$

So, if we can prove that left $A / I$-modules $A_{+} / I \underset{Z\left(A_{+}\right)}{\bigotimes_{Q}} X$ and $A_{+} / I \underset{A_{Z\left(A_{+}\right)}}{\hat{\otimes}} J$ are projective, then we shall obtain a contradiction of the estimation $d h_{A / I} X \geqslant 2$. 

The projectivity of the left Banach $A / I$-module $A_{+} / I \hat{\bigotimes}_{z\left(A_{+}\right)} X$ follows from the
existence of a morphism of left Banach $A / I$-modules

$$
\kappa: A_{+} / I \underset{z\left(A_{+}\right)}{\otimes} X \rightarrow A_{+} / I \hat{\otimes} A_{+} / I \underset{z\left(A_{+}\right)}{\bigotimes_{0}} X: \bar{a} \otimes x \mapsto \bar{a} \otimes \bar{e} \otimes x,
$$

such that $\pi_{+}^{\circ} K=1_{A_{+} /{ }_{z\left(A_{+}\right)} \hat{\otimes}^{\prime}}$, where

$$
\pi_{+}: A_{+} / I \hat{\otimes} A_{+} / I \bigotimes_{Z\left(A_{+}\right)} X \rightarrow A_{+} / I \bigotimes_{Z\left(A_{+}\right)} X: \bar{a} \otimes \bar{b} \otimes x \mapsto \bar{a} \bar{b} \otimes x .
$$

The morphism $\kappa$ is well-defined, because, by Lemma 2.4.4 of [14], for the primitive ideal $I$, we have $Z\left((A / I)_{+}\right) \simeq \mathbb{C}$, and hence

$$
\kappa(\bar{a} \cdot z \otimes x)=\bar{a} \bar{z} \otimes \bar{e}_{+} \otimes x=\bar{a} \otimes \bar{z} \bar{e}_{+} \otimes x=\bar{a} \otimes \bar{e}_{+} \otimes z . x=\kappa(\bar{a} \otimes z \cdot x)
$$

for any $z \in Z\left(A_{+}\right), \bar{a} \in A_{+} / I, x \in X$, and $\bar{z} \in \tilde{\tau}(z)$.

Since the left $\left(A, Z\left(A_{+}\right)\right)$-module $J$ is projective, there exists a morphism of left $\left(A, Z\left(A_{+}\right)\right)$-modules $\rho: J \rightarrow A_{Z\left(A_{+}\right)} \hat{\bigotimes}_{Z\left(A_{+}\right)} J$ such that $\pi_{+}{ }^{\circ} \rho=1$, where

$$
\pi_{+}: A_{Z\left(A_{+}\right)} \underset{Z\left(A_{+}\right)}{\hat{\bigotimes}} J \rightarrow J
$$

is a canonical morphism. Then the morphism of left $A / I$-modules

$$
\tilde{\rho}: A_{+} / I \bigotimes_{A_{Z\left(A_{+}\right)}} J \stackrel{1 \otimes \rho}{\longrightarrow} A_{+} / I \bigotimes_{A_{Z\left(A_{+}\right)}} A_{Z\left(A_{+}\right)} \underset{Z\left(A_{+}\right)}{\hat{\bigotimes}} J \approx A_{+} / I \underset{Z\left(A_{+}\right)}{\bigotimes_{1}} J
$$

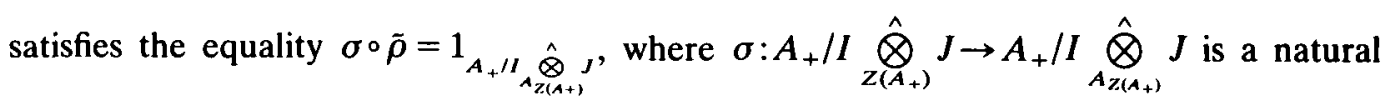
epimorphism. This shows that the left $A / I$-modules $A_{+} / I \hat{\bigotimes}_{Z\left(A_{+}\right)} X$ and $A_{+} / I{\underset{A}{Z\left(A_{+}\right)}}_{\text {projective. }} J$ are
a

For the algebra $A / I$ with an identity, we prove this statement by analogy with the previous case replacing the flat right $A_{Z\left(A_{+}\right)}$-module $A_{+} / I$ by the flat right $A_{Z\left(A_{+}\right)}$-module A/I.

Thus, by the lemma just proved, we have $d g_{Z\left(A_{+}\right)} A \geqslant 2$, and, consequently, $d b_{Z\left(A_{+}\right)} A \geqslant d g_{Z\left(A_{+}\right)} A \geqslant 2$.

\section{REFERENCES}

1. H. Cartan and S. Eilenberg, Homological algebra. (Princeton University Press, 1956). 1969).

2. J. Dixmier, Les $C^{*}$-algebras et leurs representations, 2nd ed. (Gauthier-Villars, Paris,

3. A. Ya. Helemskii, Homology in Banach and topological algebras (MSU publishers, Moscow, 1986 (Russian); English (Dordrecht; Cluwer, 1989).

4. A. Ya. Helemskii, Banach and polynormed algebras: general theory, representations, homology (Moscow, Nauka, 1989) (Russian). 
5. A. Ya. Helemskii, The periodic product of modules over Banach algebras. Funct. Analysis Appl., 5 (1971), 84-85.

6. B. E. Johnson, Cohomology in Banach algebras. Mem. Amer. Math. Soc., No 127, (1972).

7. Z. A. Lykova, The lower estimate of the global homological dimension of infinitedimensional CCR-algebras, Uspekhi Mat. Nauk, 41 (1986), 197-198 (Russian).

8. Z. A. Lykova, Structure of Banach algebras with trivial central cohomology. J. Operator Theory, to appear.

9. J. Phillips and I. Raeburn, Perturbations of $\mathrm{C}^{*}$-algebras. II. Proc. London Math. Soc. (3), 43 (1981), 46-72.

10. J. Phillips and I. Raeburn, Central cohomology of $\mathrm{C}^{*}$-algebras. J. London Math. Soc. (2). 28 (1983), 363-372.

11. J. Phillips and I. Raeburn, Voiculescu's double commutant theorem and the cohomology of C*-algebras.--preprint (University of Victoria, Victoria, British Columbia, Canada, 1989).

12. M. Putinar, On analytic modules: softness and quasicoherence, in Proc. 3-rd Conference on Complex Analysis (Varna, 1985).

13. M. A. Rieffel, Induced Banach representations of Banach algebras and locally compact groups, J. Functional Analysis, 1 (1967) 443-491.

14. C. E. Rickart, The general theory of Banach algebras (Van Nostrand, 1960).

15. Yu. V. Selivanov, Biprojective Banach algebras, lzv. Akad. Nauk SSSR ser. mat., 43 (1979), 1159-1174 (Russian).

16. J. L. Taylor, Homology and cohomology for topological algebras. Advances in Math., 9 (1972) 137-182.

Algebra and Analysis Department

Moscow Institute of Electronic Machine Building

B. VuzovskiI $3 / 12$

Moscow 109028

Russia 